Essec Business School

\title{
Approches du management de la supply chain en circuit fermé ${ }^{1}$
}

Cet article explique pourquoi et comment exploiter le remanufacturing dans le cadre du management de la supply chain en circuit fermé comme une stratégie viable afin de poursuivre un développement durable. Il montre en premier lieu l'urgence à considérer sérieusement cette problématique pour des raisons tant écologiques que légales. Il dresse ensuite un panorama des travaux existants dans le champ du remanufacturing. La revue de littérature ne se limite pas à résumer les travaux publiés suivant leur importance, mais identifie également trois axes de recherche futurs prometteurs, à savoir la gestion des acquisitions, la conception de mécanismes, et la sélection de technologie. 
ujourd'hui, la raréfaction des
matières premières et la protection
de l'environnement sont des questions de premier plan amenant tous les pays à réfléchir en termes de développement durable. Dans cet article, nous souhaitons aborder les pratiques industrielles et les approches théoriques se penchant sur ce sujet. Nous allons, dans un premier temps, souligner l'importance du développement durable et de la réutilisation des produits (remanufacturing). Nous présentons dans un second temps les travaux prééminents relatifs aux tendances en matière de réutilisation des produits. Enfin, nous formulons dans un dernier temps des suggestions quant aux domaines les plus pertinents pour de futurs travaux de recherche.

Du fait de la mondialisation, les niveaux de production atteignent des proportions auparavant inimaginables. Dans la mesure où cette croissance dépend d'un approvisionnement stable en matières premières, les entreprises multinationales commencent à se soucier de la disponibilité future des ressources naturelles, étant donné que celle-ci diminue alors que leur prix augmente constamment. Les entreprises multinationales voient donc de plus en plus la réutilisation des produits et matériaux comme une source alternative de matières premières et intègrent une production s'appuyant sur le recyclage dans leur stratégie à long terme. Xerox, par exemple, a incorporé cette réutilisation dans la production de sa gamme de photocopieurs et connaît une certaine réussite dans la mise sur le marché de cette ligne de produits remanufacturés (Debo et al., 2005). HP, Sony, Braun et Electrolux sont les membres fondateurs de la plateforme européenne de recyclage (ERP, European Recycling Platform). Cette alliance stratégique a permis à ces quatre géants de l'électronique de mettre en place un circuit de recyclage pour les équipements électriques et électroniques de leurs divisions $B$ to $B$. Les membres du réseau ERP proposent ainsi la reprise d'un matériel ancien en échange d'un modèle récent. Dans ce secteur, les entreprises englobent d'ailleurs déjà le remanufacturing dans leur stratégie de prix et de services.

Outre les raisons d'ordre économique, les questions relatives à la préservation de l'environnement ont entraîné l'apparition de mesures législatives liées à la réutilisation des matériaux et au recyclage. Par exemple, sous la houlette du gouvernement de coalition réunissant les socio-démocrates et le parti écologiste, l'administration allemande a entrepris un large éventail de réformes dont un des objectifs principaux était la réduction de la (sur)consommation des matières et des ressources énergétiques dans la production; l'idée étant que plus les ressources demeurent dans le circuit de production moins elles génèrent de déchets ou de pertes. En juin 2000, la commission européenne a présenté un certain nombre de mesures visant à gérer le problème des déchets électroniques. En 2002, ces propositions se sont traduites par des directives européennes relatives aux déchets d'équipements électriques et électroniques (directives DEEE), statuant que « la mise en œuvre d'une démarche de développement durable nécessite des changements importants dans les schémas de développement, de production et de consommation ainsi que dans les comportements, et prône la réduction du gaspillage des ressources naturelles et la protection de l'environnement ». Dans ces directives, les déchets provenant d'équipements électriques et électroniques 
(DEEE) sont cités parmi les domaines devant être régulés, en soulignant le principe de prévention, récupération et retraitement des déchets en toute sécurité. Dans la pratique, cela signifie que le traitement des DEEE relève de la responsabilité des entreprises fabriquant ces équipements et que ces dernières doivent donc mettre en place des infrastructures capables d'effectuer la collecte sélective des DEEE, afin que les « foyers utilisateurs de ces équipements aient la possibilité de restituer les DEEE sans frais supplémentaires ». Les entreprises ont également l'obligation de se charger des déchets collectés de manière écologique, à savoir en effectuant un traitement ne nuisant pas à l'environnement ou en envisageant leur réutilisation (ou refurbishing).

La réutilisation des matériaux s'avère particulièrement pertinente dans le cas de l'industrie électronique pour plusieurs raisons. La première réside dans le fait que les cycles de vie des produits électroniques se sont considérablement raccourcis, diminuant d'autant la durabilité réelle de beaucoup de produits. On peut estimer, par exemple, la durée de vie moyenne d'un téléphone mobile comme étant de cinq à sept ans, mais les statistiques montrent qu'en réalité ceux-ci sont utilisés pendant un an et demi seulement. Le dirigeant d'HP, Klaus Hieronymi confirme que « en comparaison à des machines à laver, les équipements électroniques ne sont pas jetés parce qu'ils ont cessé de fonctionner mais parce que de nouveaux appareils arrivent sur le marché ». De son point de vue, la course à la performance dans l'industrie électronique a entretenu une base de clients exigeants avides d'innovations récentes. Le rythme rapide - de ces innovations a fait le succès de ce secteur mais a néanmoins occasionné en parallèle de sérieux problèmes environnementaux. Selon des évaluations statistiques récentes, la quantité de déchets électroniques générés chaque année dépasserait 40 millions de tonnes. En 1998, l'Europe à elle seule en a produit six millions de tonnes, un volume qui croît, selon les estimations, de 3 à $5 \%$ par an. D'autre part, la production de la plupart de ces appareils électroniques est elle-même forte consommatrice de ressources, ce qui contribue à l'augmentation du prix des matières premières et de l'énergie. La production d'un ordinateur de $24 \mathrm{~kg}$ utilise environ $240 \mathrm{~kg}$ d'énergies fossiles et de produits chimiques. L'augmentation de la demande concernant certaines matières premières telles que l'acier, le plastique, l'or ou le cuivre entraîne l'augmentation des coûts de production. Les grandes firmes multinationales de l'électronique ont pris conscience d'éventuelles pénuries à venir et ont commencé à envisager la réutilisation de produits dans le processus de production comme une approche stratégique particulièrement sensée dans le futur. Cette démarche est d'ailleurs un axe majeur du développement durable. Guide (2000) fournit des données précises sur l'ampleur du remanufacturing aux États-Unis. On estime ainsi à plus de 73000 le nombre d'entreprises impliquées dans cette activité aux ÉtatsUnis, celles-ci employant plus de 350000 personnes. Ces activités engrangent des revenus supérieurs à 35 milliards de dollars par an (U.S. Environmental Protection Agency, EPA 1997, cité in Guide, 2000).

\section{Tour d'horizon de la littérature}

Le remanufacturing est une stratégie de production dont l'objectif est la revalorisation de produits usés à travers l'utilisation 
Figure 1 - Les flux de matières dans le processus de remanufacturing

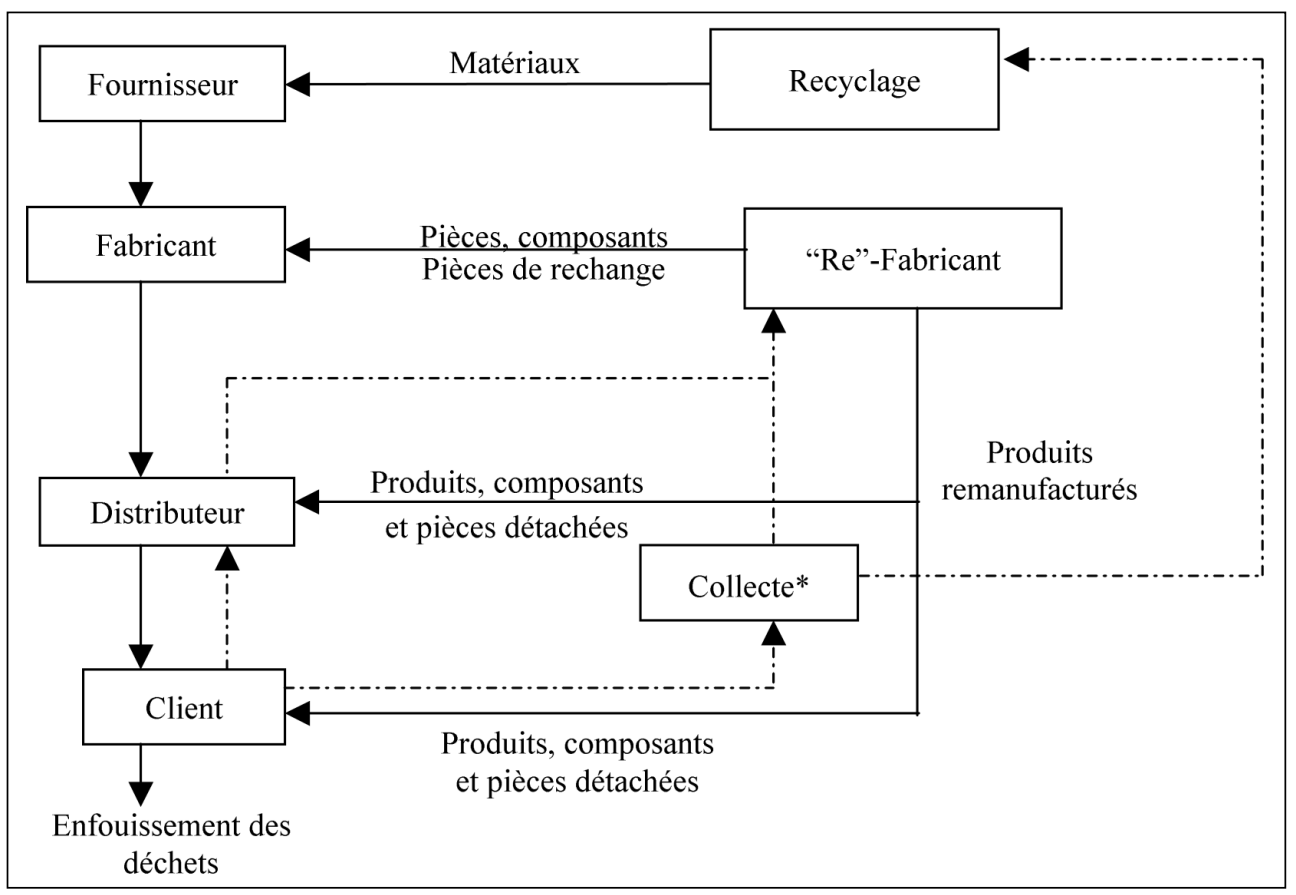

* En cas de surstocks, d'invendus et de retours sur garantie, le distributeur peut être amené à se charger de la collecte.

de composants fonctionnant correctement (Debo et al., 2005). Cette définition est illustrée dans le schéma du processus de remanufacturing proposé par Souza et al. (2001).

Après avoir proposé une description schématique mettant en lumière la complexité du processus de remanufacturing (figure 2), Guide (2001) cherche à montrer que les caractéristiques complexes de ce système doivent être optimisées conjointement plutôt que de manière indépendante.

Les produits remanufacturés sont obtenus en récupérant des produits usés et en remplaçant les pièces usagées par des pièces neuves (Thierry et al., 1995). Ce processus clôt le cycle d'utilisation des matériaux et forme un système de production en circuit fermé (Guide, 2000). Les critères et types de produits intervenant dans la faisabilité d'un tel processus ont été répertoriés par Lund (1998). Guide et Van Wassenhove (2001), pour leur part, distinguent trois axes stratégiques : la gestion de l'acquisition des produits, le développement du marché des produits remanufacturés et les problématiques spécifiques au remanufacturing. Majumder et Groenevelt (2001), quant à eux, se sont penchés sur l'étude de la concurrence dans cette activité et pensent que les OEM devraient augmenter la part de produits réutilisables disponibles ou diminuer les coûts de remanufacturing.

La plupart des études relatives à la réutilisation des produits sont assimilées à la gestion des flux. La gestion des flux est appré- 
Figure 2 - Processus de remanufacturing et flux d'information

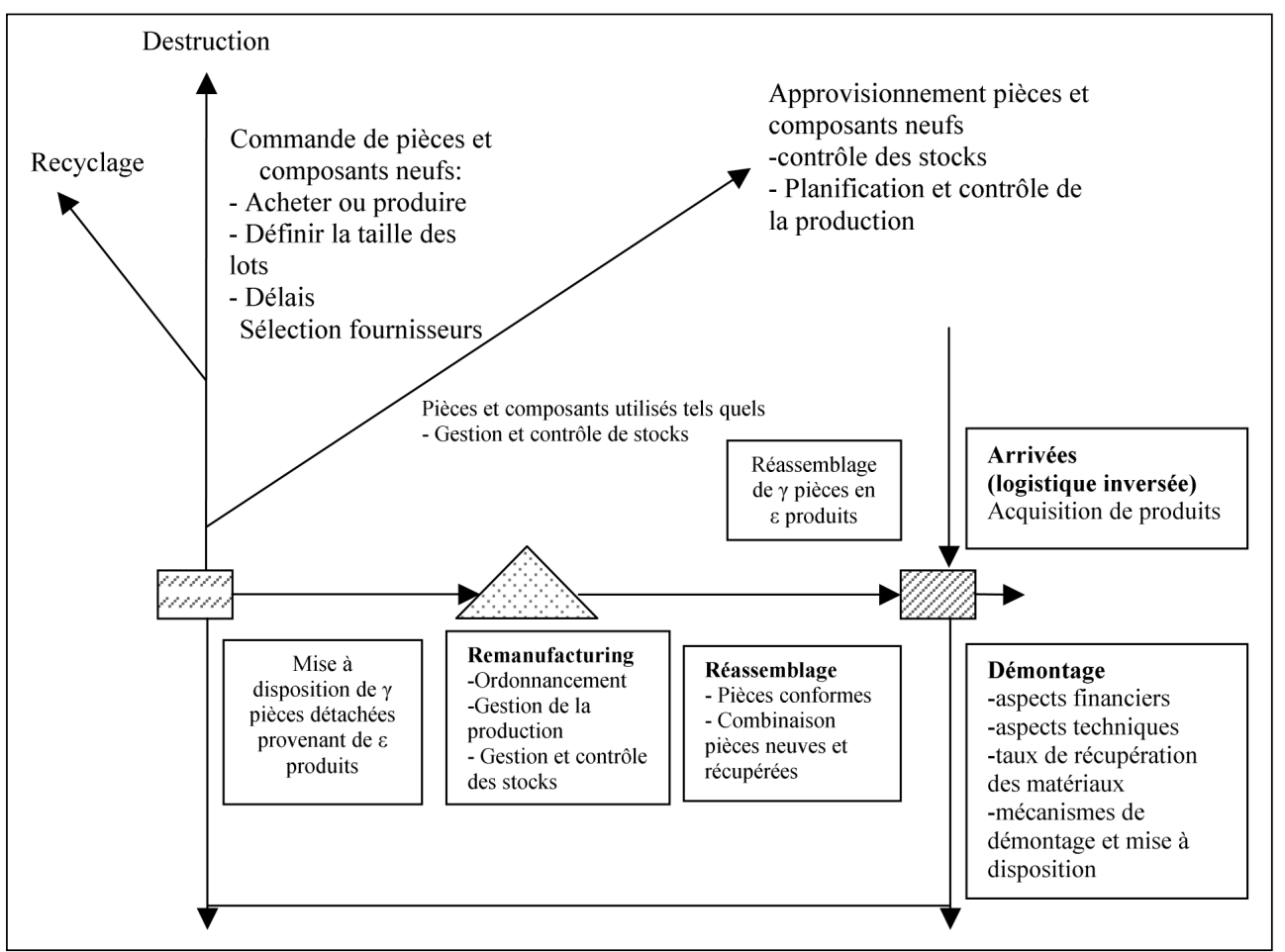

hendée à travers divers axes de recherche, notamment la prévision, la logistique inversée, l'ordonnancement la gestion des stocks. Guide (2000) propose en une synthèse particulièrement utile (tableau 1).

Dans la gestion des flux, le retour des produits est considéré comme une donnée externe, la société ré-utilisatrice étant considérée comme un acteur unique et le processus étant perçu comme déterminé. Au-delà de ces hypothèses, des études plus récentes ont suivi plusieurs grands courants: la gestion de l'acquisition des produits (les retours n'étant pas considérés uniquement comme une donnée externe), la conception des mécanismes (comprenant plusieurs acteurs dans un cadre de théorie des jeux) et la sélection des technologies (le remanufacturing pouvant être un choix endogène).

\section{La gestion des flux}

Les questions opérationnelles sont principalement liées à la gestion des stocks, au contrôle de la production et à la logistique inversée (flux remontant de produits usés). La plupart des ouvrages traitant de la gestion des flux posent l'hypothèse que le prix et le niveau de réutilisation possible sont exogènes, et que les consommateurs ne font pas la différence entre les produits neufs et les produits remanufacturés. Si ces hypothèses sont valables pour certains types de produits - l'appareil photo jetable Kodak 
Tableau 1 - Vue d'ensemble des approches relatives au management des flux

\begin{tabular}{|c|c|c|c|c|}
\hline Prévision & $\begin{array}{l}\text { Logistique } \\
\text { inversée }\end{array}$ & $\begin{array}{c}\text { Planification } \\
\text { et contrôle } \\
\text { de la production }\end{array}$ & $\begin{array}{l}\text { Gestion } \\
\text { et contrôle } \\
\text { des stocks }\end{array}$ & Général \\
\hline $\begin{array}{l}\text { Modèles } \\
\text { statistiques } \\
\text { (Goh et } \\
\text { Varaprasad, 1986) }\end{array}$ & $\begin{array}{l}\text { Étude des } \\
\text { ouvrages publiés } \\
\text { et de la structure } \\
\text { des problèmes } \\
\text { (Fleischmann } \\
\text { et al., 1997) }\end{array}$ & $\begin{array}{l}\text { Opérations } \\
\text { de démontage } \\
\text { (Brennan et al., } \\
1994 ; \\
\text { Guide et } \\
\text { Srivastava, 1998) }\end{array}$ & $\begin{array}{l}\text { Systèmes de suivi } \\
\text { périodique de la } \\
\text { demande } \\
\text { indépendante } \\
\text { (Inderfurth, } \\
\text { 1996, 1997; Richter, } \\
\text { 1996a, 1997b, 1997) }\end{array}$ & $\begin{array}{l}\text { Ferrer } \\
(1996,1997 \mathrm{a}, \\
\text { 1997b), } \\
\text { Gungor et al. } \\
(1998) \text {; Lund } \\
(1983,1998) \text {, } \\
\text { Thierry et al. } \\
\text { (1995) }\end{array}$ \\
\hline $\begin{array}{l}\text { Retours de } \\
\text { conditionnements } \\
\text { réutilisables } \\
\text { (Kelle et Silver, } \\
\text { 1989) }\end{array}$ & $\begin{array}{l}\text { Structure des } \\
\text { problèmes } \\
\text { (Flapper, 1995a, } \\
\text { 1995b, 1996; } \\
\text { Sarkis et al., } \\
\text { 1995; Driesch } \\
\text { et al., 1997) }\end{array}$ & $\begin{array}{l}\text { Aspects } \\
\text { économiques } \\
\text { du démontage } \\
\text { (Johnson et Wang, } \\
1995 \text {; Penev } \\
\text { et de Ron, 1996; } \\
\text { Lambert, 1997; } \\
\text { Zussman et al., } \\
\text { 1994; } \\
\text { Veerakamilmal } \\
\text { et Gupta, 1998) }\end{array}$ & $\begin{array}{l}\text { Systèmes } \\
\text { de suivi continu } \\
\text { (Muckstadr } \\
\text { et Isaac, 1981; } \\
\text { Van der Laan, 1997; } \\
\text { Van der Laan et al., } \\
\text { 1996) }\end{array}$ & \\
\hline \multirow[t]{3}{*}{$\begin{array}{l}\text { Récupération } \\
\text { de pièces } \\
\text { détachées } \\
\text { (Krupp, 1992) }\end{array}$} & $\begin{array}{l}\text { Conception des } \\
\text { réseaux et } \\
\text { stratégies de } \\
\text { collecte (Kroon } \\
\text { et Vrijens, 1996; } \\
\text { Krikke, 1998; } \\
\text { Jayaraman et al., } \\
\text { 1999) }\end{array}$ & 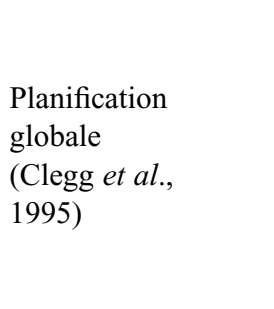 & $\begin{array}{l}\text { Fabrication } \\
\text { combinant } \\
\text { pièces neuves et } \\
\text { récupérées } \\
\text { (Salomon et al., } \\
\text { 1997; Van der Laan } \\
\text { et al., 1995, 1996, } \\
\text { 1999) }\end{array}$ & \\
\hline & & $\begin{array}{l}\text { Ordonnancement } \\
\text { et contrôle } \\
\text { de la production } \\
\text { (Guide, 1996; } \\
\text { Guide et Srivastava, } \\
1997 \text {; Guide et al., } \\
1997 \text { a, 1997b) }\end{array}$ & $\begin{array}{l}\text { Demande } \\
\text { dépendante (Flapper, } \\
\text { 1994; Gupta et } \\
\text { Taleb, 1994; Guide } \\
\text { et Srivastava, 1997; } \\
\text { Inderfurth et Jensen, } \\
\text { 1998) }\end{array}$ & \\
\hline & & $\begin{array}{l}\text { Planification de } \\
\text { la capacité (Guide } \\
\text { et Spencer, 1997; } \\
\text { Guide } \text { et al., 1997c) }\end{array}$ & $\begin{array}{l}\text { Étude } \\
\text { d'ensemble } \\
\text { (Corbey et al., } \\
\text { 1998) }\end{array}$ & \\
\hline
\end{tabular}


cité en introduction par Toktay et al. (2000) est un exemple - elles ne s'appliquent plus $\mathrm{du}$ tout si on se tourne vers l'industrie du pneu, par exemple, comme le montrent Debo et al. (2005). La gestion des flux vise à concevoir une politique de contrôle des coûts. De ce fait, on perçoit bien que la plupart des articles considèrent le remanufacturing comme un centre de coûts plutôt qu'une source de profits, même si on voit apparaître un cadre d'optimisation des profits dans certains documents plus récents (Debo et al., 2005).

Fleischmann et al. (1997), de même que Guide et al. (2000), proposent des travaux très intéressants sur la gestion des flux, nous ne reviendrons donc pas sur les recherches qu'ils ont présentées afin de nous concentrer sur des ouvrages plus récents qui n'ont pas encore été examinés. Les travaux de Van der Laan et al. (1999) étudient un système de contrôle de la production et des stocks dans un cadre à la fois de fabrication et de remanufacturing. Ils comparent les systèmes de contrôle de la production en flux poussés et en flux tirés et partagent un point de vue managérial relatif aux effets du remanufacturing sur le contrôle des stocks, en énonçant notamment des recommandations fondamentales s'adressant aux entreprises productrices de produits remanufacturés : i) contrairement à ce que l'on peut penser de manière intuitive, il peut s'avérer peu intéressant, d'un point de vue économique, de traiter tous les produits retournés, même si le niveau des retours est inférieur à celui de la demande, ii) les entreprises productrices de produits remanufacturés devraient chercher à réduire autant que possible les incertitudes en termes de délais et de qualité des retours, iii) ces entreprises devraient chercher à établir un historique des corrélations entre les retours des produits et la demande.

Toktay et al. (2000) s'attachent, en ce qui les concerne, aux processus de retour dans un système de remanufacturing. Suivant l'exemple de l'appareil photo jetable Kodak, ils estiment que l'utilisation que le consommateur fait du produit est un facteur

Figure 3 - Modèle de réseau de file d'attente

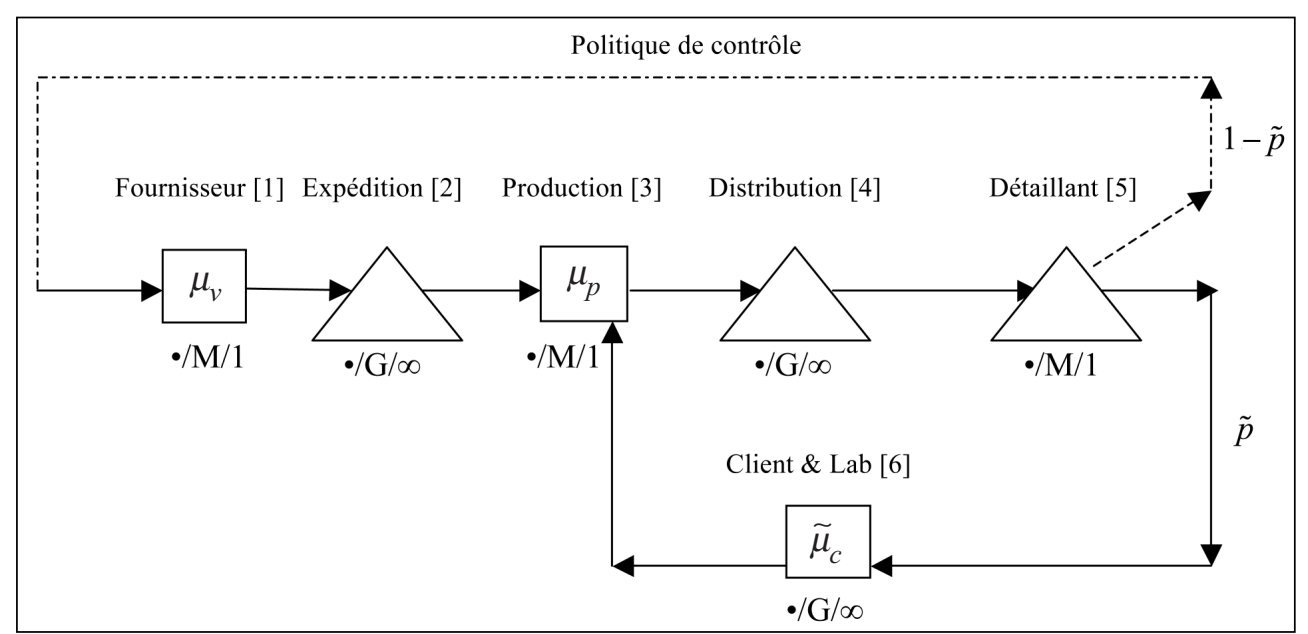


clé du système. C'est en effet une étape qui ne peut pas être observée et les délais, tout comme les taux de retour, sont aléatoires. Toktay et al. (2000) utilisent un réseau de file d'attente pour représenter le processus des flux afin de saisir sa nature aléatoire (figure 3). L'objectif du fabricant est d'optimiser la valeur actuelle nette de lancement d'un produit réutilisable, calculée sur la durée du cycle de vie du produit, en déterminant le niveau de réutilisation possible ainsi qu'une séquence de prix pour les produits neufs et pour les produits remanufacturés. Cette analyse leur permet de conclure qu'il est important d'évaluer les appareils à disposition des consommateurs mais qu'il n'est pas aussi important de les suivre.

Souza et al. (2002) se penchent sur la planification et sur le contrôle de la production dans le cas de fabricants de produits remanufacturés ayant la possibilité de vendre des articles de niveaux de qualité différents. Ils proposent un large choix de gammes de produits sur le long terme tout en veillant à maintenir la constance du niveau de service, en particulier en termes de délais. Le point important révélé ici réside dans le fait que la gamme de produits permettant d'optimiser les profits n'est pas nécessairement celle dont les marges sont les plus élevées. De plus, les règles de répartition sont un élément capital dans la réduction des délais et dans l'amélioration des niveaux de service.

\section{La gestion des acquisitions}

L'acquisition des produits dans un cadre de remanufacturing est un domaine qui possède un potentiel conséquent. Dans tous les systèmes de réutilisation des produits, les retours constituent une étape incontournable. La plupart des publications considè- rent les matériaux entrant dans le processus de remanufacturing comme un facteur externe.

L'article de Guide et al. (2003) est l'une des références principales en matière de gestion d'acquisition des produits. Selon les auteurs, l'acquisition des produits peut être dynamique et avoir un impact réel sur la rentabilité du remanufacturing. Il s'agit d'une approche de management relativement novatrice en Europe, et donc chargée de perspectives. En effet, la majorité des entreprises européennes perçoit les retours produits surtout comme une obligation légale. La figure 4 illustre le système en place actuellement en Allemagne pour répondre aux exigences des directives DEEE.

Ce système de recyclage peut être décrit de la manière suivante :

1) Depuis mars 2006, tous les produits électroniques répertoriés dans les directives DEEE ne peuvent plus être jetés dans les poubelles domestiques. Les consommateurs sont invités à apporter leurs déchets électroniques dans un des centres de collecte (Wertstoffhof) qui ont été ouverts à cet effet dans toutes les communes allemandes (Munich en a déjà 12).

Dans ces déchetteries, les DEEE sont répartis en 5 grandes catégories, chacune disposant de son propre container. La catégorie 3 , par exemple, est celle des appareils de télécommunication tels que les téléphones portables, les ordinateurs portables, les imprimantes, etc. et comprend également les appareils de « loisir » tels que les télévisions, les lecteurs DVD et les équipements hi-fi.

2) Lorsqu'un container est plein, le centre prend contact avec la fondation EAR (Elektro-Altgeräte Register), dont le rôle est de centraliser la coordination des DEEE en 
Figure 4 - Système de recyclage actuel en Allemagne, géré par une entité centrale

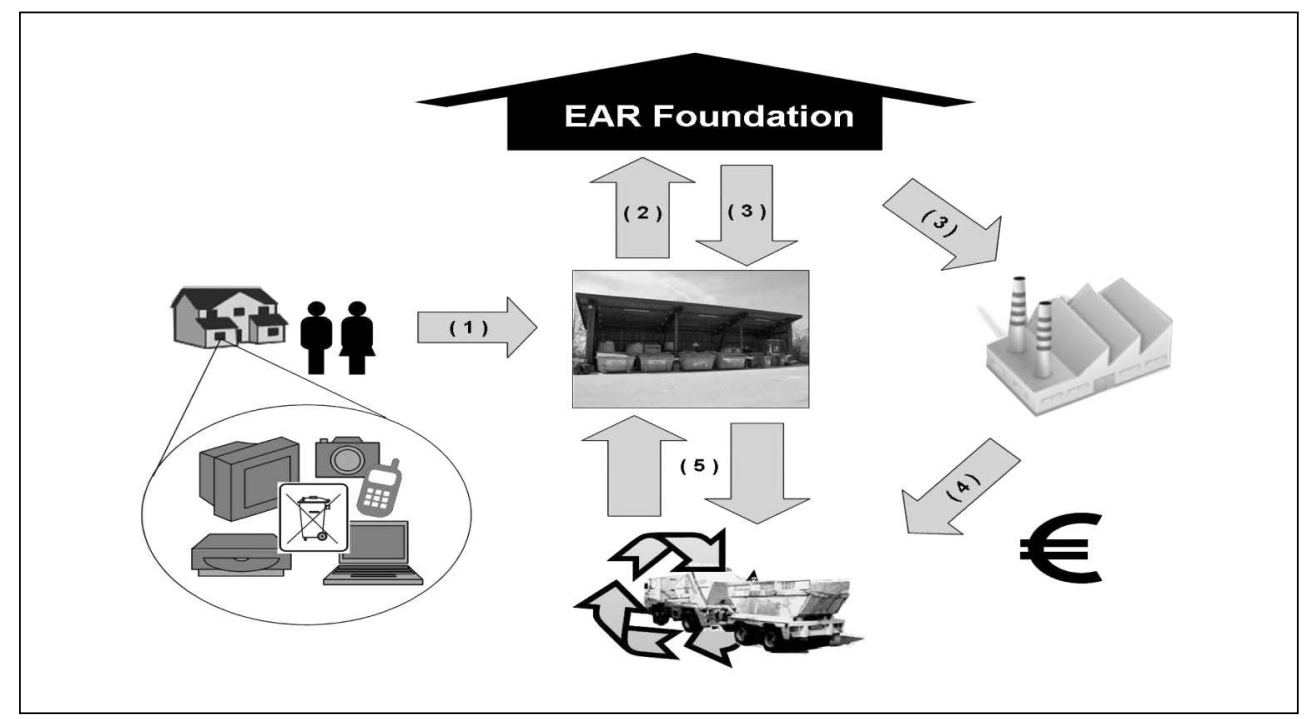

Allemagne. Tous les fabricants d'appareils électriques et électroniques en Allemagne ont l'obligation de s'inscrire auprès de la fondation EAR, à qui ils se doivent de fournir la composition de leur portefeuille de produits (les produits destinés au $B$ to $B$, ceux destinés au $B$ to $C$, ceux contenant des EEE), la durée de vie moyenne de ces produits et leur poids net. Les entreprises doivent également communiquer les chiffres de ventes de ces produits. En fonction des informations fournies, la fondation EAR attribue à chaque entreprise une part des DEEE.

3) Une fois informée par la déchetterie locale, la fondation EAR définit, à partir d'un algorithme, le container que le fabricant prendra en charge. Un numéro de routage est ensuite envoyé à la déchetterie et au fabricant concerné.

4) D'après la loi allemande sur les EEE (Elektrogesetz), le fabricant dispose de 5 jours pour venir récupérer le container et recycler son contenu. Dans la pratique, la plupart des entreprises ne se chargent pas elles-mêmes du recyclage mais emploient les services d'un spécialiste.

5) Le spécialiste du recyclage prend alors directement contact avec la déchetterie et vient récolter le container. Les matériaux utiles sont transmis ultérieurement au fabricant, après démontage.

Ce système présente un certain nombre de problèmes. En premier lieu, les consommateurs n'ont, d'un point de vue purement financier, aucun intérêt particulier à participer. Il peut leur sembler plus simple de jeter les déchets plutôt que de les amener jusqu'à une déchetterie. Un autre grand problème vient du fait que la centralisation de ce système souffre des failles typiques des organisations bureaucratiques. À l'origine, la fondation EAR s'était engagée à gérer toutes les questions directement avec tous 
les inscrits, allant même jusqu'au dialogue personnel. Cependant, lorsque l'activité a augmenté, la fréquence des requêtes en a fait de même, ce qui s'est traduit par une pression croissante sur l'institution qui, au lieu d'employer les services de centres d'appels supplémentaires, a fini par suspendre entièrement ses services en 2007 , n'étant plus en mesure de répondre aux questions administratives de tout le monde. Depuis, la section FAQ de son site web reste la seule source d'information disponible sur les actions liées aux directives DEEE. D'autre part, les déchetteries commencent à se plaindre des problèmes de délais dans le système actuellement en place, le délai moyen de collecte d'un container plein étant de 10 jours. Enfin, certaines communautés se sont vues dans l'obligation de faire surveiller les containers de DEEE face aux « attaques » de trafiquants à la recherche des matériaux les plus précieux se trouvant à l'intérieur.

Contrairement aux entreprises européennes, qui subissent quelque peu les retours de produits au regard des lois relatives à la protection de l'environnement, un certain nombre d'entreprises américaines ont choisi une approche active tournée vers le marché dans leur gestion des acquisitions de produits. Cette différence fondamentale dans la gestion des retours a d'énormes implications. D'une part, comme nous l'avons mentionné plus haut, les entreprises impliquées dans cette « remontée des déchets » considèrent cette démarche comme un centre de coûts plutôt qu'une source de profits. D'autre part, les produits constituant ces retours sont en général usagés ou de mauvaise qualité et donc, par conséquent, les possibilités de réutilisation sont limitées. Cela diminue les perspectives de remanufacturing et constitue un des grands freins à son essor en Europe. Une filiale du groupe Michelin, la société Pneu Laurent, doit ainsi acheter des lots mélangés dont la qualité est incertaine et dans lesquels il lui faut trouver suffisamment de pneus Michelin acceptables pour procéder au rechapage. Ceci pèse sur la marge et rend l'activité essentiellement déficitaire. Une approche tournée vers le marché permettrait de dynamiser le développement du remanufacturing en Europe. La mise en correspondance de l'offre et la demande de produits remanufacturés s'avère particulièrement difficile du fait de l'évolution rapide de l'offre et de la demande et du fait, également, qu'elles peuvent être situées à des endroits différents. Tout se joue donc sur le fait d'acheter les bonnes quantités, la bonne qualité, au bon prix et au bon endroit. En théorie, plus la qualité du produit retourné est basse, plus son prix est bas mais plus il est coûteux de le réutiliser. La clé du profit se trouve dans l'équilibre du coût d'acquisition et du coût de remanufacturing. La meilleure solution semble résider dans le contrôle de la qualité des produits acquis servant de matériau de base dans le processus de réutilisation. En Europe, a contrario, les entreprises sont plutôt passives vis-à-vis des « remontées de déchets » et cherchent seulement à minimiser les pertes financières.

\section{Conception des mécanismes}

Les travaux sur la conception des mécanismes se penchent sur la question de la coordination des différents facteurs internes et externes à l'entreprise afin de maximiser le potentiel de profit du remanufacturing. Savaskan et al. (2005) cherchent à comprendre l'impact que le choix du canal de distribution peut avoir sur la rentabilité du 
remanufacturing. Les auteurs établissent une comparaison entre quatre scénarios différents (figure 5) : i) le fabricant vend et collecte les produits directement auprès du consommateur, ii) le fabricant vend par le biais d'un détaillant mais collecte directement, iii) le fabricant vend et collecte par le biais d'un détaillant, iv) le fabricant vend par le biais d'un détaillant mais collecte par l'intermédiaire d'un tiers.

Dans de telles modélisations, on cherche à répondre aux deux questions suivantes:

- En quoi le prix de gros, le prix au détail et les profits de la chaîne sont-ils affectés par le choix d'une structure de logistique inversée? - En quoi les structures de supply chain en circuit fermé sont-elles susceptibles d'améliorer les taux de retours des produits et d'encourager les investissements dans la récupération de produits usagés?

Les évaluations des modèles sont données ci-dessous. Supposons que le coût unitaire de fabrication d'un nouveau produit soit représenté par $c_{m}$ et que $c_{r}$ soit le coût unitaire de remanufacturing d'un produit retourné pour en faire un nouveau produit. D'autre part, $p$ symbolise le prix de vente au détail du produit, $\phi-\beta_{p}$ est la fonction de demande du produit, $w$ le prix unitaire de gros et $b$ le prix de transfert d'un produit remontant par l'intermédiaire d'un distributeur ou d'un tiers jusqu'au fabricant. Enfin, $\mathfrak{\Im}$ est le taux de produits retournés et $C_{L}$ un paramètre constant, $\Delta$ correspond à l'économie unitaire générée par le recyclage d'un produit retourné (i.e., la différence entre $c_{m}$ et $c_{r}$ ), $A$ est le coût total de collecte et de transfert des produits retournés, et $\amalg_{j}^{i}$ désigne la fonction des profits pour les membres $j$ de la chaîne dans un modèle de supply chain $i$.

La fonction des profits et la solution optimale du modèle $\mathrm{C}$ sont formulées de la manière suivante:

$$
\begin{gathered}
\operatorname{Max}_{p, \Im} \Pi^{c}=(\phi-\beta p)\left[p-c_{m}+\Im \Delta\right]- \\
C_{L} \Im^{2}-A \Im(\phi-\beta p) \\
p^{\phi c}=\frac{\phi+\beta c_{m}}{2 \beta}-\frac{1}{2}(\Delta-A)^{2} \\
\frac{\left(\phi-\beta c_{m}\right)}{4 C_{L}-\beta(\Delta-A)^{2}} \\
\Im^{\phi C}=\frac{\left(\phi-\beta c_{m}\right)(\Delta-A)}{4 C_{L}-\beta(\Delta-A)^{2}}
\end{gathered}
$$

Figure 5 - Différentes structures de systèmes de collecte

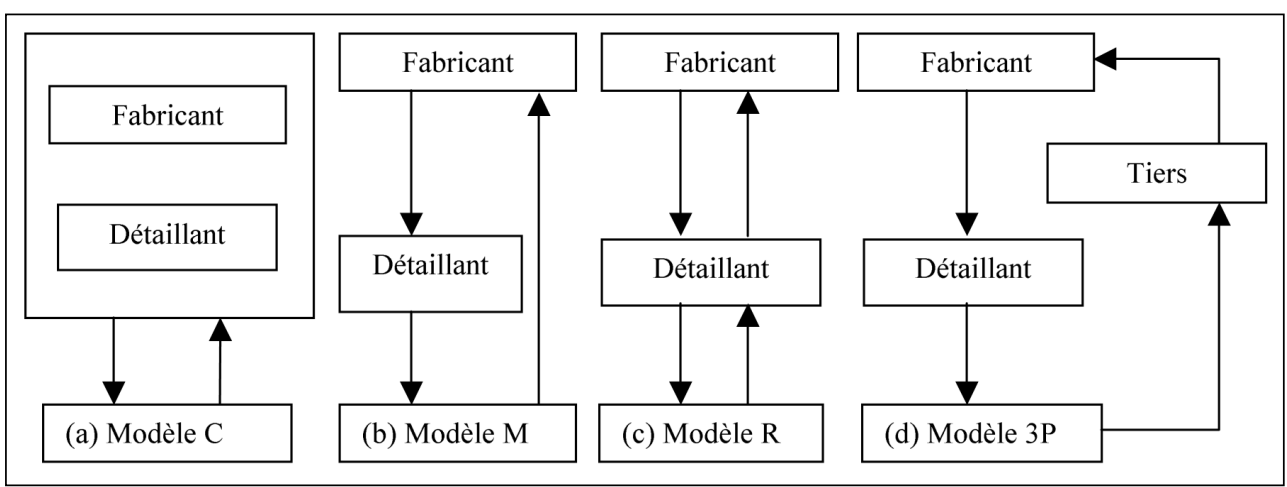


La fonction des profits et la solution optimale du modèle $\mathrm{M}$ sont formulées de la manière suivante:

$$
\begin{gathered}
\operatorname{Max}_{w, \Im} \Pi_{M}^{M}=\frac{\phi-\beta w}{2}\left[w-c_{m}+\mathfrak{\Im} \Delta\right]- \\
C_{L} \mathfrak{\Im}^{2}-A \mathfrak{s} \frac{\phi-\beta w}{2} \\
\operatorname{Max}_{w, \Im} \Pi_{M}^{M}=\frac{\phi-\beta w}{2}\left[w-c_{m}+\mathfrak{\Im} \Delta\right]- \\
C_{L} \mathfrak{\Im}^{2}-A \mathfrak{s} \frac{\phi-\beta w}{2} \\
w^{\phi M}=\frac{\phi+\beta c_{m}}{2 \beta}-\frac{(\Delta-A)^{2}\left(\phi-\beta c_{m}\right)}{2\left[8 C_{L}-\beta(\Delta-A)^{2}\right]} \\
\mathfrak{J}^{\phi M}=\frac{\left(\phi-\beta c_{m}\right)(\Delta-A)}{8 C_{L}-\beta(\Delta-A)^{2}}
\end{gathered}
$$

La fonction des profits et la solution optimale du modèle $\mathrm{R}$ sont respectivement données par:

$$
\begin{array}{r}
\operatorname{Max}_{w} \Pi_{M}^{R}=\left(\phi-\beta p^{\phi R}\right)\left[w-c_{m}+\Delta \Im^{\phi R}\right]_{-} \\
b \Im^{\phi R}\left(\phi-\beta p^{\phi R}\right)
\end{array}
$$

$\operatorname{Max}_{p \Im} \Pi_{R}^{R}=(\phi-\beta p)[p-w]+b \Im(\phi-\beta p)-$

$$
C_{L} \mathfrak{\Im}^{2}-A \Im(\phi-\beta p)
$$

$p^{\phi R}=\left(\phi+\beta\left[w-(b-A) \Im^{\phi}\right]\right) /(2 \beta)$

$\mathfrak{\Im}^{\phi R}=\left((b-A) /\left(2 C_{L}\right)\right)\left(\phi-\beta p^{\phi R}\right)$

$w^{\phi R}=\frac{\phi+\beta c_{m}}{2 \beta}-\frac{(\Delta-b)(b-A)\left(\phi-\beta c_{m}\right)}{2\left[4 C_{L}-\beta(\Delta-A)(b-A)\right]}$

Enfin, la fonction des profits et la solution optimale du modèle 3P sont respectivement données par:

$\operatorname{Max}_{\mathfrak{\Im}} \Pi_{3 P}^{3 P}=b \mathfrak{\Im}\left(\phi-\beta p^{\phi 3 P}\right)-$

$$
C_{L} \mathfrak{\Im}^{2}-A \mathfrak{\Im}\left(\phi-\beta p^{\phi 3 P}\right)
$$

$\operatorname{Max}_{w} \Pi_{M}^{3 P}=\left(\phi-\beta p^{\phi 3 P}\right)\left[w-c_{m}+(\Delta-b) \mathfrak{\Im}^{\phi 3 P}\right]$

$$
\begin{aligned}
w^{\phi 3 P}=\frac{\phi+\beta c_{m}}{2 \beta}-\frac{\phi+\beta c_{m}}{2 \beta} \\
{\left[\frac{\beta(b-A)(\Delta-b) /\left(4 C_{L}\right)}{1-\beta(b-A)(\Delta-b) /\left(4 C_{L}\right)}\right] }
\end{aligned}
$$

La comparaison des profits optimums obtenus avec les trois modèles permet d'obtenir les résultats suivants:

$$
\begin{aligned}
\mathfrak{J}^{\phi C} & >\mathfrak{\Im}^{\phi R}>\mathfrak{\Im}^{\phi M}>\mathfrak{\Im}^{\phi P} \\
\Pi_{M}^{\phi R} & >\Pi_{M}^{\phi M}>\Pi_{M}^{\phi 3 P} \\
\Pi_{R}^{\phi R} & >\Pi_{R}^{\phi M}>\Pi_{R}^{\phi 3 P} \\
\Pi^{\phi C} & >\Pi_{T}^{\phi R}>\Pi_{T}^{\phi M}>\Pi_{T}^{\phi 3 P}
\end{aligned}
$$

Cette analyse permet de tirer plusieurs conclusions :

- En premier lieu, plus un agent se trouve proche du marché, plus la collecte des produits usés est efficace pour l'ensemble des acteurs impliqués dans la chaîne. Le fabricant peut chercher à influencer la demande afin de compenser la perte de contrôle due à la décentralisation du processus.

- De plus, le prix de collecte optimum et le prix au détail optimum diminuent en fonction du niveau de décentralisation, dans la mesure où le distributeur peut agir directement sur les prix afin que ces derniers reflètent les économies unitaires réalisées.

- Enfin, la hiérarchie des taux de retour (dans l'intérêt de la société civile dans son ensemble) n'est pas toujours équivalente à la hiérarchie des profits.

Cependant, de manière générale, les mécanismes et systèmes centralisés $(\mathrm{C}) \mathrm{s}$ 'avèrent être les meilleurs. L'information capitale découlant du travail de Savaskan et al. (2005) réside dans le fait que la proximité 
du distributeur vis-à-vis du marché jour un rôle capital dans les aspects financiers du remanufacturing, d'autant plus que le distributeur a la possibilité de jouer sur le prix final en fonction des coûts unitaires, optimisant ainsi conjointement l'investissement réalisé dans la collecte de produits usés. Le fabricant, a contrario, doit tenir compte de la double marge existante, de fait, en descendant la chaîne. Faire réaliser la collecte par un tiers semble être la solution que la plupart des entreprises préfèrent. $\mathrm{Ce}$ modèle montre cependant qu'il s'agit en fait de la moins bonne solution, de tous les points de vue.

Les frais facturés par les entreprises de collecte représentent un coût direct pour la supply chain, ce qui a pour effet de diminuer la demande et la rentabilité de l'activité de remanufacturing en général. L'article de Savaskan et al. (2005) constitue une contribution intéressante dans la mesure où il étudie les interactions entre la logistique descendante et la logistique inversée, et où il modélise des formes de motivations relatives à divers acteurs au sein de différents types de structures de supply chain.
L'article de Geyer et al. (2007) s'intéresse à l'impact des facteurs internes à l'entreprise sur la rentabilité du remanufacturing: les taux de collecte, la durabilité et le cycle de vie du produit. Ces contraintes et les coûts de structure d'un système de production déterminent de manière conjointe la rentabilité du remanufacturing. Geyer et al. (2007) appuient leur démonstration relative à la durée du cycle de vie du produit et au nombre de réutilisations possibles sur l'observation de plusieurs produits différents. À propos du taux de collecte, Geyer et al. (2007) exposent, dans un premier temps, une étude détaillée des facteurs empêchant les fabricants d'effectuer la collecte des produits retournés: (a) le réseau de collecte, pour diverses raisons pratiques, ne couvre pas l'ensemble des zones dans lesquelles les produits pourraient être collectés, (b) l'utilisateur du produit, parce que cela ne lui est pas pratique ou pour d'autres considérations, peut préférer ne pas ramener le produit, (c) il existe des tiers qui collectent indifféremment toutes sortes de produits. Puis, dans un second temps, Geyer et al. (2007) présentent deux conditions fonda-

Tableau 2 - Exemples de durées de vie pour certains produits (Geyer et al., 2007)

\begin{tabular}{|c|c|c|c|c|c|}
\hline Produit & Composant & $\begin{array}{c}\text { Vie « type } \\
\text { d'un } \\
\text { composant }\end{array}$ & $\begin{array}{c}\text { Utilisation } \\
\text { «type } \\
\text { du produit }\end{array}$ & $\cap$ & Source \\
\hline Pneus voitures & Extérieur & $500000 \mathrm{~km}$ & $150000 \mathrm{~km}$ & 3 & Ferrer (1997b) \\
Ordinateurs & Processeur & $80000 \mathrm{~h}$ & $20000 \mathrm{~h}$ & 4 & Keeble (1998) \\
Appareils photo jetables & Boîtier photo & 6 cycles & 1 cycle & 6 & Kodak (1999) \\
Bouteilles verre & Produit entier & 25 cycles & 1 cycle & 25 & UBA (1996) \\
Palettes bois & Produit entier & 50 cycles & 1 cycle & 50 & UBA (1996) \\
Caissons bouteille & Produit entier & 120 cycles & 1 cycle & 120 & UBA (1996) \\
\hline
\end{tabular}


mentales pour la viabilité d'une démarche de remanufacturing. D'une part, le coût du remanufacturing doit être inférieur à celui de la production initiale. D'autre part, la valeur présente dans la possibilité de reprendre un produit usé doit être supérieure aux coûts que cette reprise implique. La contribution de Geyer et al. (2007) est intéressante en ce qu'elle modélise le cycle de vie, fini, des produits. Dans les approches antérieures, on partait, pour simplifier, du principe que tous les produits remontants peuvent être réutilisés et qu'il n'est possible de réutiliser un produit qu'une seule fois. Le modèle proposé par Geyer et al. (2007) démontre explicitement que le nombre de réutilisations possibles correspond à la durée de vie du composant divisée par la durée de vie du produit. Les auteurs soutiennent leur argumentation relative à la limite du nombre de remanufacturing et à la durée du cycle de vie du produit à partir de plusieurs produits. Afin d'optimiser les profits potentiels du remanufacturing, tous les facteurs cités plus haut doivent être articulés entre eux et avec la structure des coûts de production. Il s'agit d'un véritable défi dans la mesure où les OEM peuvent, jusqu'à un certain point, influencer tous ces facteurs. Il n'est donc pas évident de décider s'il faut les représenter comme des variables décisionnelles ou comme des paramètres. Il s'agit d'un point capital soulevé ici car les travaux de recherche antérieurs présentent tous ces facteurs comme des paramètres. En règle générale, ceux-ci impliquent une non-linéarité des coûts de production et les résultats obtenus sont donc radicalement différents des cas non contraints que l'on trouve en théorie. Enfin, la contribution la plus importante du travail de Geyer et al. (2007) tient à la démonstration de la manière dont un OEM est capable d'articuler ces nombreux facteurs afin d'augmenter la manne financière potentielle que le remanufacturing représente.

\section{Sélection de la technologie}

La plupart des publications spécialisées considèrent la possibilité du remanufacturing comme une donnée externe, alors que dans la pratique une entreprise peut influencer le potentiel de réutilisation de ses produits par l'investissement; tout simplement, par exemple, en utilisant de meilleurs matériaux pour les faire durer plus longtemps.

L'entreprise peut également améliorer cette capacité de réutilisation en investissant dans un système de traçabilité (historique électronique des données) afin de connaître l'état des composants. Il existe peu de travaux à ce sujet, l'un des plus connus étant celui de Debo et al. (2005) sur l'industrie du pneu. Le potentiel de rechapage des pneus peut évoluer en fonction des matériaux et des technologies de production choisis par le fabricant mais l'augmentation de ce potentiel passe par des coûts de production plus élevés. Dans le modèle exposé, le fabricant opte pour un certain niveau de potentiel de réutilisation en choisissant une certaine technologie de production, désignée par $q$, pour une fraction de produits pouvant être remanufacturés après un certain délai d'utilisation. Si le potentiel de réutilisation est de zéro, le produit ne peut pas être réutilisé. Le choix de la technologie a un impact sur tous les coûts initiaux - quel que soit le volume de production qui s'ensuit - tels que les dépenses de $\mathrm{R} \& \mathrm{D}$, en partant du principe que celles-ci sont un coût fixe $k(q)$ qui est une fonction convexe croissante de $q$. Un potentiel de réutilisation plus élevé nécessite 
un coût de production unitaire plus élevé et un coût de remanufacturing inférieur.

L'article de Debo et al. (2005) se distingue également dans son approche de modélisation par l'utilisation d'un modèle économique de différenciation verticale. Les consommateurs sont classés en fonction de leur disposition à payer: $F(\theta)=1-(1-\theta)^{k}$, dans un intervalle $[0,1]$. Les consommateurs considèrent que les produits remanufacturés ont moins de valeur que les produits neufs $P_{N}$.

Le prix des produits neufs et remanufacturés détermine leur demande relative pour chaque période:

$$
S=\left[\left(P_{N}, P_{R}\right) \in R_{+}^{2}: 0 \leq P_{N} \leq 1 ; 0 \leq P_{R} \leq \eta\left(P_{N}\right)\right]
$$

Il s'agit d'un problème à horizon de planification infini impliquant plusieurs autres facteurs de complexité uniques à ce modèle.

En effet, la combinaison de produits neufs et remanufacturés génère un portefeuille de produits unique en son genre dans la mesure où les produits remanufacturés n'existent que du fait de la vente, au préalable, de produits neufs. Ensuite, le fait de fabriquer des produits porteurs d'un potentiel de réutilisation peut inciter des « refabricants " indépendants à entrer en concurrence avec le fabricant initial sur cette partie spécifique du marché. Trois questions principales relatives à la sélection de la technologie émergent donc:

- La production d'un produit réutilisable est-elle rentable?

- Quelles pièces devrait-on récupérer?

- La possibilité d'atteindre les consommateurs composant cette partie spécifique du marché compense-t-elle le coût élevé des produits réutilisables?
Dans le modèle suivant, l'objectif du fabricant est de maximiser la valeur nette actuelle de mise sur le marché d'un produit réutilisable, calculée sur le cycle de vie du produit, en déterminant le potentiel de réutilisation et une séquence de prix pour les produits neufs et les produits remanufacturés. Il s'agit donc d'un problème de maximisation du profit à horizon infini en temps discret, soit:

$$
\max _{q \in[0,1]} V_{\beta}(q)-k(q)
$$

où les revenus sont définis par

$$
V_{\beta}=\max _{\Im \in(0)} \sum_{t=0}^{\infty} \beta^{t} \pi\left(v_{t}, q\right)
$$

Dans la solution optimale, le fait d'augmenter le volume de produits neufs sur une seule période entraîne une augmentation immédiate des revenus générés par ces produits ainsi qu'une augmentation des revenus générés par les produits remanufacturés sur la période suivante. Le potentiel de remanufacturing augmente lorsque la fabrication ou le remanufacturing du produit sont moins coûteux ou lorsque le marché s'appauvrit. Un marché de masse de consommateurs au pouvoir d'achat peu élevé constitue un volume d'acheteurs potentiels à satisfaire en leur proposant des produits remanufacturés.

Si les produits neufs peuvent générer des profits, ils représentent également, à terme, un volume de produits usés générant à leur tour des profits, une fois remanufacturés. D'ailleurs un fabricant peut choisir de fabriquer certains produits du fait de la valeur qu'ils représentent dans la perspective de leur vente en tant que produits remanufacturés, même s'ils sont initialement vendus à perte. Un coût de remanufacturing 
Tableau 3 - Facteurs déterminant la rentabilité du remanufacturing

\begin{tabular}{|c|c|c|}
\hline $\begin{array}{c}\text { Paramètre, } \\
\text { croissant }\end{array}$ & Interprétation & $\begin{array}{c}\text { Impact sur } \\
\text { le potentiel }\end{array}$ \\
\hline$C_{r} \mathrm{cr}(0)$ & Coût de remanufacturing & négatif \\
$k^{\prime}(0)$ & Augmentation des coûts fixes & négatif \\
$B$ & Facteur d'escompte/Laps de temps passé sur le marché & positif \\
$A$ & Dépréciation perçue & négatif \\
$c^{\prime}(0)$ & Augmentation des coûts unitaires de production & négatif \\
$c_{a}(0)$ & Coût de production pour une seule utilisation & positif/négatif \\
$K$ & Profil consommateur & négatif \\
\hline
\end{tabular}

Source : Debo et al. (2005).

bas peut permettre un niveau de potentiel de réutilisation supérieur et un volume alors plus important de produits réutilisables pourra alors être vendu de manière optimale. Un coût de remanufacturing bas peut donc se traduire aussi bien par une augmentation que par une baisse des ventes de produits neufs, ce qui est difficile à prévoir.

Le tableau 3 résume l'impact de tous les facteurs sur la rentabilité du remanufacturing.

\section{Conclusion}

Cet article souligne l'importance du supply chain management en circuit fermé et du remanufacturing dans un contexte de développement durable et montre qu'ils peuvent constituer une activité génératrice de profits. Les fabricants seront de plus en plus amenés, dans le futur, à mettre en place de telles démarches, que ce soit pour répondre au marché ou aux exigences législatives. Il semble donc intéressant de s'y préparer dès maintenant. Ce domaine est à l'origine de nombreux sujets de recherche; nous avons donc répertorié les écrits les plus impor- tants et identifié de futurs axes de recherche envisageables.

Cette étude a commencé autour de la gestion des flux, sujet qui a été largement étudié. Il représente donc des possibilités de recherche nouvelles fort limitées, qui nécessiteraient elles-mêmes une analyse au préalable de toutes les publications existantes afin d'éviter la répétition de contributions préexistantes. De plus, tout nouveau travail éventuel sur le sujet ne pourrait apporter qu'une contribution marginale aux travaux existants et exigerait des cadres mathématiques plus que poussés. C'est pourquoi peu d'articles ont été publiés récemment sur le sujet.

Les trois autres axes de recherche, à savoir la gestion des acquisitions, la conception des mécanismes et la sélection de la technologie, sont a contrario relativement nouveaux et ont relativement fait l'objet d'un nombre limité de publications jusqu'à présent. Ils présentent donc des possibilités beaucoup plus ouvertes, pouvant faire l'objet de nouveaux articles et pour lesquelles il est aisé d'éviter les répétitions. Les paru- 
tions récentes relatives au supply chain management en circuit fermé dans des revues de haut niveau couvrent d'ailleurs essentiellement ces sujets. L'acquisition de produits en s'appuyant sur le marché semble particulièrement pertinente pour les entreprises européennes qui ne voient pour le moment les produits en fin de vie que comme des obligations et non comme des opportunités, contrairement à leurs homologues américaines.
Enfin, si le développement du marché des produits remanufacturés fait l'objet de nombreuses discussions en termes de législation et de prise de conscience de la part des consommateurs, il n'existe pas de modèle d'investissement dans les produits remanufacturés et de dynamique de marché les concernant. Ceci représente donc un champ d'exploration particulièrement intéressant pour le futur.

\section{Bibliographie}

Debo L., Toktay B. et van Wassenhove L.N., "Market segmentation and product technology selection for remanufacturable product", Management science, vol. 51, 2005, p. 11931205.

Fleischmann M., Bloemhof-Ruwaard J., Dekker R., van der Laan E., van Nunen J.A.E.E. et van Wassenhove L.N., "Quantitative models for reverse logistics: A review", European journal of operational research, vol. 103, 1997, p. 1-17.

Geyer R., van Wassenhove L.N. et Atasu A., "The Economics of remanufacturing under limited component durability and finite product life cycles", Management science, vol. 53, p. 88-100, 2007.

Guide V.D.R., "Production planning and control for remanufacturing: Industry practice and research needs", Journal of operations management, vol. 18, 2000, p. 467-483.

Guide V.D.R., Harrison T.P. et van Wassenhove L.N., "The challenge of closed-loop supply chains", Interfaces, vol. 33, 2003a, p. 3-7.

Guide V.D.R., Jayraman V., Srivastava R. et Benton W.C., "Supply chain management for recoverable manufacturing systems", Interfaces, vol. 30, 2000, p. 125-142.

Guide V.D.R., Teunter R.H. et van Wassenhove L.N., "Matching supply and demand to maximize profits from remanufacturing", Manufacturing and service operations management, vol. 5, 2003b, p. 303-316.

Guide V.D.R. et van Wassenhove L.N., Business aspects of closed-loop supply chains, Carnegie Mellon University Press, Pittsburgh, 2003.

Guide V.D.R. et van Wassenhove L.N., "Managing product returns for remanufacturing", Production and operations management, vol. 10, 2001, p. 142-154.

Lund R., "Remanufacturing: An american resource", Proceedings of the $5^{\text {th }}$ International Congress on Environmentally Conscious Design and Manufacturing, June 16-17, Rochester Institute of Technology, Rochester, NY, 1998. 
Majumber P. et Groenevelt H., "Competition in remanufacturing", Production and operations management, vol. 10, 2001, p. 125-141.

Savaskan R.C., Bhattacharya S. et van Wassenhove L.N., "Closed-loop supply chain models with product remanufacturing”, Management science, vol. 50, 2004, p. 239-252.

Souza G., Wagner H.M. et Whybark D.C., "Evaluating focused factory benefits with queuing theory”, European journal of operational research, vol. 128, 2001, p. 597-610.

Souza G., Ketzenberg M. et Guide V.D.R., "Capacitated remanufacturing with service level constraints", Production and operations management, vol. 11, 2002, p. 231-248

Thierry M., Salomon M., van Nunen J. et van Wassenhove L.N., "Strategic issues in product recovery management", California management review, vol. 37, 1995, p. 114-135.

Toktay L.B., Wein L.M. et Zenios S.A., "Inventory management of remanufacturable products", Management science, vol. 46, 2000, p. 1412-1426.

van der Laan E., Salomon M., Dekker R. et van Wassenhove L.N., "Inventory control in hybrid systems with remanufacturing", Management science, vol. 45, 1999, p. 733-747. 